\title{
Sociedad Española de Cerámica y Vidrio. Sus primeros pasos.
}

\author{
ANTONIO GARCÍA VERDUCH \\ Universidad Jaume I. Instituto de Cerámica y Vidrio. CSIC.
}

Conferencia de Clausura del XL Congreso Nacional de la Sociedad Española de Cerámica y Vidrio

Onda (Castellón), del 8 al 11 de Noviembre de 2000

\section{1.- INTRODUCCIÓN}

Los miembros de la Comisión Organizadora de este cuadragésimo Congreso Nacional me han rogado que atraiga vuestra atención hacia aquellos tiempos fundacionales de la Sociedad Española de Cerámica y Vidrio, que tan intensamente compartí con un puñado de entrañables compañeros. $Y$ precisamente, el haber vivido con tal intensidad tan feliz episodio me incapacita para hacer un relato desapasionado. La historia nunca la hacen los protagonistas, sino aquellos que, mucho tiempo después, tienen la habilidad y la paciencia de rebuscar en las cenizas.

Por estas razones, me limitaré a ofreceros dos estampas, la primera de ellas sobre los antecedentes de la Sociedad, su constitución y sus primeros balbuceos, y la segunda, sobre el espíritu que animó su fundación, y sobre el papel que desempeñó en el modelado de un nuevo pensamiento.

\section{2.- HURGANDO EN LA PREHISTORIA}

El Instituto de Edafología y Fisiología Vegetal, del Consejo Superior de Investigaciones Científicas, estaba trabajando ya activamente a mediados de los años cuarenta, y a finales de ese decenio tenía su edificio propio en la calle de Serrano, de Madrid, muy cerca de la sede central del C.S.I.C. Su director era D. José María Albareda Herrera, su vicedirector D. Lorenzo Vilas López, y su secretario, D. Tomás Alvira Alvira. De la Sección de Físico-Química de Suelos se encargaba D. Vicente Aleixandre Ferrandis. En esa Sección comencé los estudios sobre arcillas, que dieron lugar a mi tesis doctoral, concluida a principios del año 1951. Poco tiempo después, tomando como núcleo esa Sección, se constituyó el Departamento de Silicatos, de más amplio alcance, que dependía del Patronato "Juan de la Cierva" de Investigación Técnica.

En ese Departamento de Silicatos, alojado en el mismo edificio del Instituto de Edafología, y dirigido por D. Vicente Aleixandre Ferrandis, se realizaron estudios abiertamente relacionados con otras ramas tecnológicas. Allí se hicieron las tesis doctorales de Demetrio Alvarez-Estrada, sobre cerámica de esteatita, de Jaime Robredo Olave, sobre tierras de moldeo y, posteriormente, de José María Fernández Navarro, sobre vidrios. Desde este Departamento se iniciaron todos los contactos con personas, con empresas y con instituciones, que dieron lugar al nacimiento de la Sociedad Española de Cerámica, en el año 1960, y del Instituto de Cerámica y Vidrio, en el año 1970.

Puede decirse, con plena justicia, que durante las dos fértiles décadas comprendidas entre 1950 y 1970, el Departamento de Silicatos del C.S.I.C. jugó un papel decisivo en la implantación del pensamiento científico en los sectores cerámico y vidriero de España.

Los últimos meses de 1958 tuvieron una significación muy especial en la gestación de la Sociedad Española de Cerámica, porque fué entonces cuando se produjo la feliz coincidencia de los factores positivos, que voy a enumerar:

1.- $\quad$ El Departamento de Silicatos, en razón de su propio trabajo, había tenido ya numerosos contactos con el mundo industrial, y había palpado la inquietud que producían las deficiencias técnicas propias, cuando las empresas miraban hacia el futuro. Además, las noticias que llegaban del extranjero, relacionadas con nuevos avances, hacían aumentar dicha inquietud.

2.- $\quad$ En el Departamento de Silicatos coincidíamos por entonces, Demetrio Alvarez-Estrada, ya doctor, que había realizado trabajos de investigación en Suecia; Jaime Robredo Olave, ya doctor, que había hecho una larga estancia de trabajo científico en Holanda; José García Vicente, también doctor, especialista en cristalografía, y otros varios doctores del Instituto de Edafología, que habían hecho investigaciones sobre arcillas en diversos países europeos; y yo mismo, que había trabajado en el Imperial College of Science and Technology, de Londres, y en el Instituto de Silicatos, de Gotemburgo, Suecia y, después, había hecho una larga permanencia en los Estados Unidos, investigando en el Instituto Tecnológico de Massachusetts y en la Universidad de Alfred, de Alfred, New York. Además, y ésto es muy importante, contábamos con la sabiduría, la profunda experiencia y las magníficas relaciones de nuestro director, D. Vicente Aleixandre Ferrandis. 
3.- La biblioteca del Departamento de Silicatos había recibido por aquellas fechas una donación de colecciones de revistas cerámicas, formada por unos 260 volúmenes, que había gestionado yo durante mi estancia en la Universidad de Alfred, N.Y., de los Estados Unidos. Esta donación llegó como agua de mayo en el desierto de nuestros recursos bibliográficos relacionados con la cerámica.

4.- El Instituto de Edafología y Fisiología Vegetal vió, con naturalidad y simpatía, el hecho de que en su propio seno se gestase una importante iniciativa, que iba a redundar en beneficio de unos campos tan distintos del suyo.

5.- Con el fin de tantear la opinión externa al Departamento de Silicatos, acerca de la creación de una sociedad científica de cerámica, se remitieron dos o tres centenares de cartas de exploración a posibles interesados, y de las impresiones recibidas, unas fueron alentadoras y otras abiertamente entusiastas.

6.- Otra circunstancia muy favorable era que el minúsculo grupo de promotores de una asociación cerámica, nos hallábamos integrados en las plantillas de la investigación oficial, y que, por tanto, vivíamos de nuestros sueldos y no poseíamos intereses en el sector industrial. Por esa razón, nuestras voces eran desinteresadas, y no despertaban ningún recelo en el mundo de la producción.

$\mathrm{Y}$ así, poco a poco, fueron encajando, unas en otras, todas las circunstancias favorables que acabamos de mencionar, y ese feliz acoplamiento no señalaba más camino que el de comenzar a actuar.

La fundación de la Sociedad Española de Cerámica no se produjo como fruto de un largo proceso de debate, ni como resultado de ninguna negociación. Todo ocurrió de un modo tan simple como el desprendimiento del árbol de una fruta que está ya madura. Simplemente, tenía que ocurrir y ocurrió.

\subsection{El modo de agruparse}

Cuando todo ésto ocurría, a finales de 1958, la Asociación Nacional de Químicos estaba en el proceso de constituir en su seno unas Secciones Técnicas especializadas, que tenían por objeto estimular los contactos y la mutua colaboración entre todos los químicos interesados en cada sector. Así, por ejemplo, si la memoria no me falla, se habían constituido ya las de Cosmetología y Corrosión.

Dado que los miembros del Departamento de Silicatos éramos todos químicos, y afiliados a nuestra Asociación Nacional, parecía obligado considerar, en primer lugar, la posibilidad de crear una Sección Técnica de Cerámica en el seno de la Asociación Nacional de Químicos.

Después de una reunión que mantuvimos los miembros del Departamento de Silicatos con otros compañeros del sector industrial - entre los que se hallaban Cipriano Coma Díaz y Joaquín Pibernat Lleyxá - decidimos renunciar a ese modo de agrupación, que tanto habría simplificado los trámites y el papeleo. La razón fué así de sencilla: El centro de gravedad de nuestras inquietudes no se hallaba en la química, sino en la cerámica.

En nuestro ánimo no estaba la limitación de la cerámica a sus aspectos puramente químicos. Deseábamos abarcarla en su totalidad, sin descartar ninguno de sus matices. Si dábamos el paso de constituir una Sección Técnica de Cerámica dentro de la Asociación Nacional de Químicos, no debería extrañarnos que los físicos, los ingenieros $u$ otros grupos profesionales, acabasen constituyendo en el seno de sus
Asociaciones análogas Secciones Técnicas, lo cual conduciría a una situación caótica, absolutamente indeseable, y muy dañina para los intereses del sector cerámico.

Después de estos tanteos y consideraciones, quedó ya perfectamente claro en nuestra mente cuál habría de ser el modo de agrupamiento. Deseábamos una Sociedad Española de Cerámica abierta a todas las personas interesadas, que abarcase todos los materiales cerámicos existentes y por crear, y todos los conocimientos que, de un modo $\mathrm{u}$ otro, tuviesen relación con ellos. Queríamos una Sociedad así de universal, tan abierta al pasado histórico como al futuro prometedor, $\mathrm{y}$ en la cual cupiesen todas las personas, de dentro o de fuera, que quisieran sumar sus conocimientos a los nuestros.

Esta decisión dejaba muy claro que lo que había que hacer inmediatamente era comenzar a trabajar: Redactar proyectos de Estatuto y Reglamento, acopiar información acerca de los trámites a realizar, hacer los contactos personales necesarios para configurar una comisión organizadora oficial y una propuesta de la primera Junta Directiva. Y después, convocar la obligada Asamblea de constitución, y seguir haciendo trámites para dejar perfectamente atornillada la estructura de la nueva Sociedad Española de Cerámica, y su fundamento legal.

\section{3.- CONSTITUCIÓN DE LA SOCIEDAD ESPAÑOLA DE CERÁMICA}

Antes de comenzar cualquier tramitación, había que buscar un nombre para la Sociedad, para una Sociedad que aspiraba a ser de ámbito nacional. Para ello se hizo una consulta a la Oficina de Información de la Presidencia del Gobierno, sobre el uso de los términos "nacional" y "española". El 21 de Enero de 1959 se nos comunicó que el uso de "española" era libre, pero el de "nacional" requería una autorización expresa de la Presidencia del Gobierno. Así, pues, quedaba expedito el camino para llamar a nuestra sociedad "Sociedad Española de Cerámica".

Una vez decidida la denominación de la nueva Sociedad, se procedió a la redacción de unos Estatutos que, con fecha 20 de Febrero de 1959, se remitieron a la superioridad, para su aprobación, acompañados de una instancia en la que se justificaba la creación de una Sociedad de esta naturaleza. En ella se decía "que es deseo de un amplio sector de la industria cerámica española y de los investigadores científicos de esta especialidad, el agruparse en una Sociedad que tenga como última meta la mejora del nivel científico y técnico de esta rama de la industria española". También se añadía, como apoyo, que "para que la cerámica española se vea oficialmente representada en la Asociación Europea de Cerámica, es requisito indispensable la existencia de una Sociedad Española de Cerámica de características análogas a las de las Sociedades de los otros países miembros".

Esta solicitud de aprobación de los Estatutos, la firmamos D. Vicente Aleixandre Ferrandis, D. Francisco Arredondo y Verdú, y yo mismo, como miembros de la Comisión Organizadora encargados de hacer los trámites necesarios para la fundación de la Sociedad Española de Cerámica.

Los restantes miembros de la Comisión Organizadora eran D. Florentino Gómez Ruimonte, D. Cipriano Coma Díaz, D. Demetrio Alvarez-Estrada, D. Eugenio Azcárraga Vela, D. José García Vicente, D. Luis Gómez Centurión, D. Jaime Robredo Olave y $\mathrm{D}^{\mathrm{a}}$ Piedad de la Cierva Viudes, y actuaba como Secretario de dicha Comisión D. Antonio García Verduch. 


\section{1.- Asamblea Fundacional}

En contestación a nuestra solicitud del 20 de Febrero de 1959, se recibió una notificación, fechada el 18 de Junio de 1959, en la cual la Dirección General de Política Interior, del Ministerio de la Gobernación, daba su aprobación a los Estatutos de la Sociedad Española de Cerámica.

Una vez aprobados los Estatutos de la Sociedad, ya quedaba vía libre para convocar una reunión de las personas simpatizantes, con el fin de proceder a la constitución formal de la Sociedad Española de Cerámica y a la designación de su primera Junta Directiva.

La Comisión Organizadora pensó celebrar esa importante reunión en un lugar digno, como era el Salón de Actos del Patronato "Juan de la Cierva" de Investigación Técnica, situado en la planta baja del edificio de ese Patronato, situado en la calle Serrano $n^{\circ} 150$, de Madrid. Por entonces, el edificio era casi nuevo, y su Salón de Actos, con capacidad para unas 150 personas, era realmente acogedor. En la convocatoria se fijo la reunión para el día 16 de Febrero de 1960, a las 18,30 h.

El día 14 de Enero de 1960 se dirigió un escrito a D. Manuel Lora Tamayo, que entonces era Secretario General del Patronato "Juan de la Cierva" de Investigación Técnica, solicitando autorización para utilizar ese Salón de Actos, y el 20 de Enero nos contestó con un oficio en el que se decía: “... tengo el gusto de comunicarle que pueden disponer del Salón de Actos de este Patronato, el día 16 de Febrero próximo para celebrar la reunión de constitución de la Sociedad Española de Cerámica".

Así, pues, ya teníamos un proyecto de Reglamento y una propuesta de primera Junta Directiva, que había que discutir en la asamblea de constitución. Además, teníamos un texto de Estatuto aprobado por la superioridad, y el permiso para la utilización del Salón de Actos. La convocatoria para el acto había sido enviada ya a muchos posibles asistentes. Sólo faltaba que, a aquel hermoso salón de actos, viniese alguien interesado en fundar una sociedad que se llamaría Sociedad Española de Cerámica.

Aquella tarde del 16 de Febrero de 1960, con mucha anticipación a la hora señalada para la reunión, ya estábamos en aquel edificio, nuestro compañero Demetrio Alavez-Estrada, propuesto para el cargo de Tesorero, y yo, como Secretario de la Comisión Organizadora, para cuidar de que todo estuviese a punto.

El salón tenía sus puertas abiertas y sus luces encendidas, y nosotros nos mirábamos con preocupación viendo cómo se iba acercando la hora, y la asistencia seguía siendo aún escasa. ¡Y cuál fué nuestra sorpresa al ver que, en los últimos minutos, acudían nutridos grupos de personas, muchas de las cuales eran desconocidas para nosotros!. A la reunión acudieron unas setenta personas, la mayoría de las cuales eran residentes en provincias, que habían venido ex profeso a este acto de fundación de la Sociedad. En esos instantes comprendimos que, a la seriedad de nuestra convocatoria, habían correspondido muchas personas con la seriedad de su asistencia.

La reunión comenzó bajo la presidencia de D. Vicente Aleixandre Ferrandis, acompañado en la mesa por D. Francisco Arredondo y Verdú, y por mí mismo, actuando los tres como representantes de la Comisión Organizadora.

Después de las palabras de bienvenida, se pasó a discutir el proyecto de Reglamento presentado, y las interesantes sugerencias que iban siendo aportadas por los asistentes. Finalmente, se acordó aprobar el texto del Reglamento, con inclusión de las modificaciones indicadas.

Llegado el momento de discutir y aprobar las cuotas que habían de satisfacer los socios numerarios y los corporativos, el que iba a ser Tesorero de la Sociedad, D. Demetrio AlvarezEstrada, con cierta timidez, sugirió las cantidades de 150-200 ó 250 pts. anuales, para los socios numerarios, y de 1000-1500 ó 2000 pts. anuales para los corporativos. A la vista de estas cantidades propuestas, intervino uno de los asistentes para decir que si esta Sociedad estaba llamada a hacer cosas importantes, difícilmente podría hacerlas con unas cuotas mezquinas. Este sentir se hizo general, y enseguida se aprobaron las cuotas más altas propuestas, es decir, las de 250 pts. y 2000 pts. respectivamente.

A continuación se procedió a la elección de la primera Junta Directiva de la Sociedad, la cual quedó configurada del siguiente modo:

Presidente: D. Luis Auguet Durán Vicepresidente: D. Tomás Trénor Azcárraga, Marqués del Turia

Secretario: D. Vicente Aleixandre Ferrandis

Vicesecretario: D. Antonio García Verduch

Tesorero: D. Demetrio Alvarez-Estrada SECCIÓN DE CIENCIA BÁSICA

Presidente: D. José I. Fernández Alonso

Vicepresidente: D. Juan Luis Martín Vivaldi

Secretario: D. José García Vicente

SECCIÓN DE PRODUCTOS DE ARCILLA

Presidente: D. Luis Gómez Centurión

Secretario: D. Francisco Arredondo y Verdú

SECCIÓN DE PORCELANAS

Presidente: D. Juan Giralt Esteva

Vicepresidente: D. Alberto Alvarez González

Secretario: D. Jaime Robredo Olave

SECCIÓN DE REFRACTARIOS

Presidente: D. José María de Bilbao Arístegui

Vicepresidente: D. Bartolomé Darnís Bellido

Secretario: D. Florentino Gómez Ruimonte

SECCIÓN DE ARTE CERÁMICO

Presidente: D. Jacinto Alcántara Gómez

Vicepresidente: D. Carlos de Miguel González

Secretario: D. Cipriano Coma Díaz

COMISIÓN PERMANENTE DE EDUCACIÓN

Presidente: D. Enrique Gutiérrez Ríos

Vicepresidente: D. Alfonso Blat Monzó

Secretaria: Da. Piedad de la Cierva Viudes

Vocales: D. José María Costa Serrano

D. Víctor de Nalda Frigols

Al ser aprobada la nueva Junta Directiva, D. Vicente Aleixandre Ferrandis invitó al Presidente electo D. Luis Auguet Durán y al Vicepresidente electo D. Tomás Trénor Azcárraga, Marqués del Turia, a ocupar la mesa presidencial. A partir de ese momento, la presidencia de la sesión pasó a D. Luis Auguet Durán.

En esta primera reunión de la Sociedad, además de adoptarse los acuerdos básicos propios de ocasiones tan singulares, se trataron otros asuntos que habían de condicionar fuertemente los designios de la Sociedad a lo largo de su vida. Merece la pena señalar algunos de ellos.

D. Vicente Aleixandre Ferrandis señaló la necesidad de estimular la investigación científica en áreas de interés cerámico, y de disponer de técnicos de nivel superior, con formación específica en esta rama de la tecnología.

A propuesta de D. José Terraza Martorell y de D. Tomás Trénor Azcárraga, Marqués del Turia, se aprobó incluir en el 
Reglamento la posibilidad de crear Delegaciones o Secciones locales o regionales de la Sociedad Española de Cerámica.

D. Francisco Arredondo y Verdú sugirió el establecimiento de la categoría de Socios Fundadores, entendiendo por tales todas aquellas entidades o personas que ingresen en la Sociedad en calidad de socios corporativos o numerarios en el periodo comprendido entre la reunión de constitución de la Sociedad y la primera Junta General de la misma.

D. Florentino Gómez Ruimonte propuso que las Juntas Generales de la Sociedad se celebren en distintas ciudades españolas.

D. Vicente Aleixandre Ferrandis razonó la conveniencia de que la Sociedad se adhiera a la Asociación Europea de Cerámica, y explicó las gestiones preparatorias que ya se habían realizado a este respecto. A petición del Presidente D. Luis Auguet Durán, la asamblea acordó continuar las gestiones y hacer el estudio económico necesario para que dicha adhesión a la Asociación Europea de Cerámica pueda producirse en breve plazo.

D. Tomás Trénor Azcárraga, Marqués del Turia, señaló la necesidad de facilitar la cooperación de los Centros de investigación, entre sí y con la industria, y de establecer enseñanzas de cerámica en niveles académicos superiores.

D. Antonio García Verduch, Vicesecretario electo, expuso la conveniencia de que la Sociedad inicie la publicación de un Boletín, que contribuiría grandemente a la consecución de uno de sus fines primordiales, que es la difusión de los conocimientos cerámicos. Este Boletín habría de tener un carácter eminentemente técnico y científico. Después expuso el resultado de un estudio económico preliminar en el que se estima que una publicación trimestral de este género costaría unas cien mil pesetas anuales. Esta propuesta fué aceptada plenamente, y se acordó que la Junta Directiva de la Sociedad la siga elaborando y llegue a proponer soluciones concretas de financiación.

D. José Terraza Martorell se interesó por la organización de la bibliografía cerámica.

Sin más asuntos que tratar, y dando como constituida la Sociedad Española de Cerámica, y elegida su primera junta Directiva, se levantó la sesión a las veinte horas del mencionado día dieciséis de Febrero de mil novecientos sesenta. De todo lo cual, como Secretario de la Comisión Organizadora, Doy Fé. Antonio García Verduch. $V^{\circ} B^{\circ}$ El Presidente de la comisión Organizadora, Vicente Aleixandre Ferrandis.

La tramitación continuó el día 19 de Febrero de 1960 elevando a la superioridad una certificación del acta de constitución de la Sociedad, y una solicitud para su inscripción en el Registro Provincial de Asociaciones, y para la legalización de los Libros de Actas, de Caja y de Socios.

Una vez constituida la Sociedad, se inició un activísimo periodo de puesta en marcha, que incluyó el establecimiento de las Delegaciones Regionales, las gestiones para iniciar la publicación del Boletín, los contactos con la Asociación Europea de Cerámica relacionados con nuestra solicitud de adhesión, el establecimiento de relaciones con otras Sociedades de Cerámica, la consolidación de la economía de la sociedad y la ampliación del número de asociados y, por supuesto, el comienzo de las actividades técnicas.

\section{2.- Delegaciones regionales}

Las Delegaciones regionales de la Sociedad quedaron al cargo de las siguientes personas:

D. Leopoldo Arche Hermosa: $\underline{\text { Santander }}$
D. Fernando Dominguez: Sevilla, Córdoba, Cádiz y Huelva

D. Juan L. Martin Vivaldi: Jaén, Granada, Málaga y Almería

D. José Lomba González: Galicia

D. José Rivas Sánchez: Asturias

D. José María de Bilbao Arístegui: Alava y Guipúzcoa

D. José Ramón Castillo Villaamil: Vizcaya

D. Luis Dalmau Castells: Cataluña

D. Tomás Trénor Azcárraga: Valencia, Castellón y Alicante

\section{3.- Publicación del Boletín}

El 2 de Febrero de 1961 se elevó una instancia al Director General de Prensa, solicitando la autorización para publicar el BOLETIN DE LA SOCIEDAD ESPAÑOLA DE CERAMICA, la cual fué concedida el 27 de Febrero de 1961. El primer número correspondió al bimestre Septiembre - Octubre de 1961, y se imprimió en los talleres de Industrias Gráficas España, S.L. de Madrid. El Director del Boletín era D. Vicente Aleixandre Ferrandis, el Gerente y Administrador, D. Demetrio Alvarez-Estrada y el Redactor-Jefe, D. Antonio García Verduch. El cuadro de redacción estaba formado por D. José García Vicente, D. Jaime Robredo Olave, D. Demetrio Alvarez-Estrada, $D^{a}$ Piedad de la Cierva Viudes, D. Francisco Arredondo y Verdú y D. Cipriano Coma Díaz.

\section{4.- Primeras actividades técnicas}

En el periodo que medió entre la constitución de la Sociedad, el 16 de Febrero de 1960, y la publicación del primer número de su Boletín, en Septiembre de 1961, se celebraron las siguientes actividades técnicas:

\section{7 de Mayo de 1960}

Conferencia-coloquio en Madrid del Prof. Joseph A. Pask, Director del Departamento de Tecnología de Minerales y Profesor de Ingeniería Cerámica de la Universidad de California.

20 a 25 de Junio de 1960

Primera Semana de Estudios Cerámicos, en colaboración con el Departamento de Silicatos del Patronato "Juan de la Cierva" de Investigación Técnica. Madrid. Doce conferencias y un curso práctico.

\section{4 de Junio de 1960}

Conferencia, en Madrid, del Prof. Claude Horan, de la Universidad de Hawai.

29 de Noviembre - 3 de Diciembre de 1960

Segunda Semana de Estudios Cerámicos, en colaboración con el Departamento de Silicatos del Patronato "Juan de la Cierva" de Investigación Técnica. Madrid. Diez conferencias y un curso práctico.

21 a 23 de Marzo de 1961

Primera Reunión Técnica de la Sección de Refractarios de la Sociedad Española de Cerámica, en colaboración con la Escuela Técnica Superior de Ingenieros Industriales de Bilbao, y los Laboratorios de Ensayos e Investigación Industrial "L. José de Torróntegui Ibarra". Cinco conferencias y visitas a los mencionados Laboratorios, al tren de bandas de Altos Hornos de Vizcaya, S.A., y a la Compañía Anónima Basconia, en Basauri.

24 a 29 de Abril de 1961

Primeras Jornadas de Arte Cerámico, en colaboración con 
la Escuela de Cerámica de Madrid. Cursillo teórico-práctico, con diversas visitas guiadas por especialistas.

1 de Mayo de 1961

Primera Conferencia de Estudios Cerámicos, VillalongaSangenjo (Pontevedra), en colaboración con la Agrupación de Fabricantes de Materiales Cerámicos de la provincia de Pontevedra. Cuatro conferencias y visitas a fábricas de ladrillos y de porcelanas.

\section{5 a 8 de Julio de 1961}

Tercera Semana de Estudios Cerámicos, Madrid, en colaboración con la Exposición Permanente e Información de la Construcción (EXCO), el Instituto Técnico de la Construcción y del Cemento (C.S.I.C.) y el Departamento de Silicatos del Patronato "Juan de la Cierva" de Investigación Técnica. Ocho conferencias y visitas a EXCO y al Instituto Técnico de la Construcción y del Cemento.

\section{5.- Socios fundadores}

En la mañana del día 4 de Julio de 1961 tuvo lugar en el salón de actos del Patronato "Juan de la Cierva" de Investigación Técnica, el solemne acto de entrega de diplomas y medallas a los Socios Fundadores de la Sociedad Española de Cerámica. La Sociedad cuenta con ciento sesenta y cinco Socios Fundadores, de los cuales ciento diez son numerarios y cincuenta y cinco corporativos.

\section{6.- Admisión de la Sociedad Española de Cerámica como miembro de la Asociación Europea de Cerámica}

La Sociedad Española de Cerámica fué admitida como miembro de la Asociación Europea de Cerámica (A.E.C.) en el año 1961. Con fecha 2 de Noviembre de 1961, el Secretario de la A.E.C. Mr. Gerald H. Steward, dirigió una carta a nuestro Presidente, en la que se decía:

"En la última reunión del Comité Directivo de la Asociación Europea de Cerámica se estudió la solicitud de admisión enviada por la Sociedad Española de Cerámica. Se acordó por unanimidad que, a reserva de la aprobación por la Asamblea de Delegados, la Sociedad Española de Cerámica sea elegida como miembro".

"Con el fin de acelerar la tramitación de admisión, se pidió por correo la opinión de los Delegados Nacionales de las organizaciones miembros, y ahora tengo el placer de informarle que la decisión del Comité Directivo ha sido confirmada por unanimidad. La Sociedad Española de Cerámica es, por tanto, ya una organización miembro de la Asociación Europea de Cerámica"-

La Asamblea de Delegados, celebrada en Copenhague el día 25 de Mayo de 1962, ratificó por unanimidad la admisión de la Sociedad Española de Cerámica como organización miembro de la A.E.C.

En representación de la Sociedad Española de Cerámica, fueron aceptados en el Comité directivo de la Asociación Europea de Cerámica D. Vicente Aleixandre Ferrandis y D. Antonio García Verduch, quienes hicieron ya acto de presencia en la reunión de dicho Comité, celebrada en Copenhague el día 24 de Mayo de 1962. En esa reunión, el Presidente Mr. G.N. Hodson dió la bienvenida a la Sociedad Española de Cerámica en términos muy cordiales, y D. Antonio García Verduch agradeció la amable acogida con unas breves palabras, de las cuales entresacamos estos dos párrafos:

"La Sociedad Española de Cerámica es el miembro más joven de esta comunidad cerámica, y por ser joven debe concentrarse en su propia consolidación y crecimiento. Ello hace prever que durante algunos años estará muy necesitada de vuestra guía y ayuda fraternales".

"Es cierto que la Sociedad Española de Cerámica siente la urgente necesidad de mirar hacia dentro, pero no es menos cierto que nosotros, los ceramistas españoles, deseamos y estamos prestos para aceptar nuestra parte de responsabilidad dentro de la A.E.C. Es evidente que nuestro desarrollo y consolidación internos han de aumentar notablemente nuestra capacidad de colaboración con esta comunidad cerámica en la que ahora ingresamos".

La delegación española designada para representar a nuestra Sociedad en la Asamblea de Delegados de la A.E.C. estuvo constituida por D. José María Pertierra, D. Alberto Alvarez González, D. Vicente Aleixandre Ferrandis y D. Antonio García Verduch.

\section{7.- Ampliación de la Sociedad al campo del vidrio}

En la Asamblea General de la Sociedad Española de Cerámica, celebrada en Sevilla el 12 de Mayo de 1967, se aprobó por unanimidad la propuesta de crear una Sección de Vidrios en el seno de la misma, y posteriormente, en la Asamblea General que tuvo lugar en Madrid el 14 de Diciembre de 1971, se acordó ampliar la denominación de la Sociedad para abarcar también el vidrio, de un modo explícito. La nueva denominación aprobada fué, pues, la de Sociedad Española de Cerámica y Vidrio y, en consecuencia, la del Boletín pasó a ser Boletín de la Sociedad Española de Cerámica y Vidrio.

El día 15 de Enero de 1972 se solicitó al Registro de la Propiedad Industrial la inscripción de la marca correspondiente a la nueva denominación del Boletín, y el 25 de Enero de 1972 se comunicó a la Dirección General de Prensa la nueva denominación de la Sociedad, el cambio de domicilio a Arganda del Rey (Madrid) y el nuevo formato del Boletín $(21 \times 29,7 \mathrm{~cm}$.)

Con toda esta tramitación, quedó el vidrio definitivamente incorporado a la Sociedad Española de Cerámica, para configurar una Sociedad ampliada en su ámbito, pero fiel a su mismo espíritu fundacional. Esta ampliación al campo del vidrio acercó a nuestra Sociedad -ya en el año 1972- a las sociedades de materiales que iban emergiendo.

\section{4.- LA SOCIEDAD ESPAÑOLA DE CERÁMICA, TRANS FORMADORA DEL PENSAMIENTO}

Los fines de la Sociedad están claramente expresados en el $\operatorname{Art}^{\circ} 2^{\circ}$ de su Estatuto: "Fomentar el avance técnico de las industrias de cerámica y de vidrio, y estimular su crecimiento y mejora mediante la investigación científica básica y aplicada, y la difusión de los conocimientos. Otro de sus fines es el de alentar las enseñanzas de cerámica y de vidrio en España".

Estos eran unos firmes deseos, que solamente podrían echar raíces y fructificar sobre un sustrato industrial receptivo y maduro. Ese sustrato existía ya en algunas industrias punteras, que poseían una mentalidad acorde con los nuevos tiempos, pero, desgraciadamente, muchas otras estaban aún ancladas en unos modos de pensar y actuar muy lejanos de la modernidad.

El firme propósito que animó a la Sociedad, desde el comienzo de su andadura, fué el de construir un nuevo pensamiento, de corte científico, que impregnase los ambientes 
industriales de la cerámica y del vidrio, y que abriese de par en par las puertas de la colaboración entre la ciencia y la industria.

La industria somnolienta estaba experimentando los primeros síntomas de asfixia. Algo que debía circular no circulaba. Las puertas estaban cerradas. Ese algo sutil e impalpable era la ciencia. La ciencia es algo vivo que necesita circular. Es una corriente impetuosa que se origina por el aporte de numerosas fuentes, y que luego riega y fertiliza. No se deben aislar esas fuentes para beber de ellas con exclusividad. Hay que dejarlas en libertad para que unan sus caudales y luego, conjuntamente, pasen por las ruedas de todos los molinos.

La Sociedad Española de Cerámica vió claramente, desde el primer momento de su existencia, que su función debía ser la de un potente equipo de bombeo, que hiciese circular los conocimientos hasta alcanzar los más finos capilares de la anatomía industrial.

Para realizar esta importante función, la Sociedad puso en marcha todos los mecanismos a su alcance para difundir la palabra hablada y la escrita, y muy pronto comenzaron a multiplicarse las reuniones técnicas y las publicaciones.

Las reuniones, además de activar la difusión de los conocimientos, cumplieron otra importantísima función, que es la de propiciar las relaciones personales necesarias para configurar una gran familia humana. La Sociedad tuvo siempre la convicción de que solamente podrían compartirse los bienes intelectuales, cuando el afecto y la cordialidad fuesen por delante abriendo los caminos. Ahora, al cabo de cuarenta años de trabajo en común, nos reafirmamos en aquella convicción nuestra de los tiempos fundacionales.

Tengo la satisfacción de manifestar a $\mathrm{Vds}$. que algunos de los viejos amigos nos legaron, como preciada herencia, la amistad de sus hijos, y posteriormente, éstos nos han dejado la de los suyos. Aquellos jovencitos que asistían con sus padres a las Reuniones Técnicas de la Sociedad, para aburrirse con la conversación de los mayores, después de muchos años -convertidos ya en magníficos profesionales y empresarios - son los que vienen ahora, acompañados de sus hijos mozos, que están haciendo sus primeras armas en la empresa o en las aulas universitarias.

\section{5.- BOLETIN DE LA SOCIEDAD ESPAÑOLA DE CERAMICA Y VIDRIO.- EL ROMPEHIELOS}

En el mes de Septiembre de 1961 salió a la luz el primer número del Boletín. Hoy, después de 39 años ininterrumpidos, se sigue publicando con regularidad. Entonces tenía un modesto contenido técnico y científico, y una formidable carga de ilusión por crear un futuro más luminoso. Hoy ya se ha hecho realidad aquel soñado futuro, y el Boletín, despojado por completo de su plumón infantil, puede codearse dignamente con las más prestigiosas publicaciones adultas de su ámbito.

No quisiera detenerme ni un instante en describir cómo se ha ido produciendo el milagro de su desarrollo, ni en señalar el ahincado esfuerzo de tantos colegas que, año tras año, han volcado en él sus ilusiones y su clarividencia. Ahora quisiera comentar solamente la función de rompehielos que cumplió el Boletín durante sus primeros años de existencia. Una publicación nueva, editada por una Asociación nueva, que pretende crear un espíritu nuevo, en una colectividad que aspira a un futuro mejor, ha de ejercer, necesariamente, una función de rompehielos.
El contenido técnico y científico de los primeros números del Boletín fué modesto, porque entonces todo era modesto. El presupuesto era limitadísimo, el apoyo humano para llevar adelante esa publicación, también lo era. Además, había poco que decir, porque los estudios y las investigaciones eran aún muy escasas en nuestro país. Todo era pequeño y modesto, como los núcleos, pero la vitalidad y el entusiasmo eran grandes.

El ambiente somnoliento que frenaba el acceso de nuestras industrias a los nuevos conocimientos, exigía una acción vigorosa y enérgica, que pudiera ser mantenida durante años y años, sin aflojar, y actuando siempre en la misma dirección. Esa es la acción de rompehielos que ejerció el Boletín, con su abundante información actualizada, con las opiniones de personas prestigiosas $\mathrm{y}$, sobre todo, con los editoriales que fueron abriendo cada número, durante los primeros ocho años de vida del Boletín.

Creemos que puede tener algún valor histórico lo que se decía en aquellos editoriales, como expresión de las inquietudes de una época. Por eso vamos a comentar el contenido de algunos de los que aparecieron en los primeros números.

$\mathrm{N}^{\mathrm{o}}$ 1.- "Aprender, enseñar". Septiembre, 1961

Había llegado el momento de emprender la gran tarea de sumar los conocimientos de todos. Todos tenían mucho que enseñar y mucho que aprender. La recién creada Sociedad Española de Cerámica estaba llamada a ser una gran escuela, donde todos debían ser maestros de lo que sabían y, a su vez, aprendices de lo que ignoraban. La transmisión oral se haría por medio de las reuniones técnicas de todo tipo, y la escrita, a través de libros, monografías, y también del Boletín que abría sus páginas por primera vez, con el editorial que llevaba este título.

Aquel primer número, de pequeño formato, constaba de 38 páginas, pero ¿cuántos miles de páginas inmaculadas quedaban esperando ser escritas en un futuro repleto de años?. Hoy, en el año 2000, contemplamos ya 39 volúmenes alineados en la estantería.

$\mathrm{N}^{\circ}$ 2.- "Metalurgia y cerámica". Noviembre, 1961

La metalurgia y la cerámica son dos ramas de la ciencia y de la técnica que se entrelazan en multitud de ocasiones, que se complementan y que se auxilian mutuamente. Y es habitual que ambas se encuentren abrazadas en el horno durante los momentos más difíciles de sus vidas. Son compañeras inseparables, compañeras de infortunio.

Al hablar de metalurgia, no hablamos solamente de metales, sino que hablamos también de escorias, de gases y vapores. Los problemas metalúrgicos son complicados, y los cerámicos también, pero las interacciones a alta temperatura entre los sistemas metalúrgicos y los cerámicos, aún encierran mayor complejidad. Para el estudio de la química de alta temperatura, deben sentarse en mesa redonda los metalurgistas y los ceramistas. Son muchos los problemas que esperan su resolución del esfuerzo conjunto de unos y de otros, y mucha es también la fertilidad de esos estudios comunes para crear nuevos materiales metalcerámicos.

En este segundo editorial, ya se hacía un guiño a la metalurgia, y se propugnaba un acercamiento a la misma para calar en las bases científicas comunes, y para abordar nuevos desarrollos tecnológicos.

$\mathrm{N}^{\circ}$ 3.- "¿Hasta donde llega nuestro oficio?". Enero, 1962

En este editorial se señalaba la necesidad de revisar el contenido y la extensión del concepto clásico de la cerámica. En esos años, la imaginación había irrumpido ya de forma arrolladora en el ámbito cerámico, y se hacía inevitable bailar al 
son de los nuevos ritmos. Se habían descubierto insospechadas propiedades de los productos cerámicos, nuevas composiciones de carácter no tradicional, nuevos procedimientos, técnicas y métodos, y nuevas materias primas naturales y sintéticas. Cuando las temperaturas o las presiones utilizadas, no eran suficientes, se elevaban, y cuando era necesario, se combinaban las altas presiones y las temperaturas elevadas.

La cerámica había desbordado ya todas sus definiciones, y avanzaba por nuevos derroteros. La cerámica ya no era lo que todos creíamos que era, y se había convertido dócilmente en aquello que nosotros queríamos que fuese. La ciencia de los materiales, con todo su sentido actual, estaba ya en la mente y en los propósitos de aquella naciente Sociedad Española de Cerámica de 1962. "La cerámica llega hasta donde nosotros queramos llevarla. Su potencialidad no se agota". Esto decíamos en 1962. Y ahora, 38 años más tarde, seguimos pensando lo mismo.

$\mathrm{N}^{\circ}$ 4.- "Producción y uso de los materiales". Marzo, 1962

Es frecuente que los artículos que salen al mercado, para el consumo del gran público, estén compuestos por diversos materiales, como cerámica, metales, vidrios, plásticos, adhesivos, pinturas, etc. Cada uno de esos materiales ha sido llamado a su puesto de servicio, porque ha podido acreditar que posee unas propiedades que son las deseadas. El buen funcionamiento del conjunto depende, por una parte, del correcto acoplamiento de los componentes, y por otra, de la calidad e idoneidad de los materiales empleados.

Todo ello viene a demostrar que no se concibe un uso adecuado de los materiales, si no se conocen sus propiedades con detalle y, en consecuencia, el modo de fabricarlos para que tengan precisamente esas propiedades, y no otras.

Para lograr el éxito global, no basta con que los materiales tengan las propiedades que deben tener. Es necesario también que se haga el uso adecuado de cada material. De nada sirve fabricar con meticulosidad unos materiales, si luego se usan con ligereza. Hay que conocer para fabricar y hay que conocer para usar. El resumen de todos los resúmenes es que hay que conocer. Esa es la única salida.

$N^{\circ}$ 5.- "De cara a Europa". Mayo, 1962

Este año, por primera vez, se ha escuchado la voz de los ceramistas españoles en el Comité Directivo de la Asociación Europea de Cerámica (A.E.C.). Una placa en bronce con el nombre de España y una bandera roja y gualda han presidido la mesa de la delegación española en Copenhague. Nuestra bandera está ya alineada con las del resto de países miembros de la A.E.C.

Los países fundadores de la A.E.C., y los que inmediatamente se unieron a ella, han tendido ahora su mano fraternal a los ceramistas españoles. Ese apretón de manos recibido es la firma y la rúbrica de un compromiso formal de cooperación.

Es cierto que nuestra Sociedad es la más joven e inexperta de todas las que integran la A.E.C. No lo decimos con rubor, sino con la clara conciencia de lo que tiene de impulso y de esperanza la savia joven. La preocupación esencial de la Sociedad Española de Cerámica ha de ser hoy su propia consolidación. Cuanto más robusta sea, más útil será a sí misma, y con más decoro y eficacia podrá cumplir sus responsabilidades europeas.

$\mathrm{N}^{\circ}$ 6.- "Primum vivere...". Julio, 1962

Primero vivir y después filosofar. Primero atender las urgentes necesidades inmediatas, y después lo demás. Lo urgente es hacer y después, si queda tiempo, pensar. Lo inmediato absorbe, ciega y esclaviza. Si uno espera pacientemente a que llegue una feliz ocasión que le brinde tiempo libre para pensar, verá con desilusión que nunca llega. La rutina y el martilleo diario, lleno siempre de premuras, llegan a moldear una conducta y una forma de ser que no permiten mirar al futuro, ni evaluar la experiencia del pasado, ni casi vivir el presente.

En cualquier actividad productiva han de participar las fuerzas del pensar y las del hacer. Las primeras siempre precediendo a las segundas.

Cuando los cuadros de personal de una empresa son esqueléticos, y se cargan sobre las personas demasiadas exigencias de tareas apremiantes, los brazos aceleran su ritmo, pero las cabezas se sumergen poco a poco en una neblina imperceptible, pero viscosa. $Y$ se va y se viene por los mismos caminos. Se trillan esos caminos más y más, porque no hay luz que alumbre otros nuevos. ¡Y a ese trillar y trillar se le llama ganar experiencia. Sí, ganar mucha experiencia, ahondando siempre las mismas carrileras!

¡Cuántas empresas anquilosadas existen, que no progresan porque están demasiado ocupadas haciendo las cosas mal, y no tienen tiempo para pensar en nuevos caminos!. Nadie tiene tiempo, ni el de abajo ni el de arriba, porque todos han de compartir la urgente tarea de producir. Producir para vender, y vender para comer. Y sigue la rueda. 